\title{
Reliability of a lifetime history of major depression: implications for heritability and co-morbidity
}

\author{
D. L. FOLEY, M. C. NEALE AND K. S. KENDLER ${ }^{1}$ \\ From the Departments of Human Genetics and Psychiatry at the Virginia Institute for Psychiatric and \\ Behavioral Genetics, Medical College of Virginia/Virginia Commonwealth University, Virginia, USA
}

\begin{abstract}
Background. In unselected samples, the diagnosis of major depression (MD) is not highly reliable. It is not known if occasion-specific influences on reliability index familial risk factors for MD, or how reliability is associated with risk for co-morbid anxiety disorders.
\end{abstract}

Methods. An unselected sample of 847 female twin pairs was interviewed twice, 5 years apart, about their lifetime history (LTH) of MD, generalized anxiety disorder (GAD) and panic disorder (PD). Familial influences on reliability were examined using structural equation models. Logistic regression was used to identify clinical features that predict reliable diagnosis. Co-morbidity was characterized using the continuation ratio test.

Results. The reliability of a LTH of MD over 5 years was fair $(\kappa=0 \cdot 43)$. There was no evidence for occasion-specific familial influences on reliability, and heritability of reliably diagnosed MD was estimated at $66 \%$. Subjects with unreliably diagnosed MD reported fewer symptoms and, if diagnosed with MD only at the first interview, less impairment and help seeking, or, if diagnosed with MD only at the second interview, fewer episodes and a longer illness. A history of co-morbid GAD or PD is more prevalent among subjects with reliably diagnosed MD.

Conclusions. A diagnosis of MD based on a single psychiatric interview incorporates a substantial amount of measurement error but there is no evidence that transient influences on recall and diagnosis index familial risk for MD. Quantitative indices of risk for MD based on multiple interviews should reflect both the characteristics of MD and the temporal order of positive diagnoses.

\section{INTRODUCTION}

Epidemiological studies commonly assess a subject's risk for major depression (MD) on the basis of a single psychiatric interview. Given that the accuracy of diagnostic assignments predicate the accurate characterization of familial-epidemiological risk factors for MD, the reliability with which non-clinical subject's recall past episodes of MD is of considerable importance. In community samples, kappa $(\kappa)$ for the test-retest reliability of a lifetime history (LTH) of MD ranges between 0.21 and 0.87

${ }^{1}$ Address for correspondence: Dr Kenneth Kendler, Virginia Institute for Psychiatric and Behavioral Genetics, Department of Psychiatry, PO Box 980710, Richmond, VA 23298-0710, USA.
(Prusoff et al. 1988; Williams et al. 1992; Keller et al. 1995). In samples comprising patients and their relative, $\kappa$ ranges between 0.61 and 0.85 (Prusoff et al. 1988; Fendrich et al. 1990; Rice et al. 1992). These reliability estimates indicate that the diagnosis of a LTH of MD is not highly reliable, especially in epidemiological samples.

Two previous investigators have examined the characteristics of reliably diagnosed MD within a genetically informative framework. Rice et al. (1992) conducted two personal interviews with a selected sample of 1629 first-degree relatives of 187 bipolar, 78 bipolar II and 331 MD probands. Six year stability of the Schedule for Affective Disorders and Schizophrenia (SADS) depressive disorder diagnosed following Research Diagnostic Criteria (RDC) 
(Spitzer et al. 1975) was good $(\kappa=0 \cdot 61)$. Covariates of stable diagnostic assignments were assessed using a forward prediction paradigm that used a diagnosis of depression at time 1 to identify a positive case. The depressive features at time 1 that predicted that depression was diagnosed at time 2 were an increasing number of depressive symptoms and hospitalization or treatment with medication or electroconvulsive therapy.

Kendler et al. (1993) subsequently examined the reliability of a LTH of MD in an unselected sample of 1721 female twins. Twins were initially surveyed by a mailed questionnaire that included five of the nine DSM-III-R (American Psychiatric Association (APA), 1987) MD symptoms and a LTH of MD was assigned if any three symptoms, including depressed mood, had co-occurred for at least 2 weeks. Approximately 1 year later, a LTH of MD was assessed again using a face-to-face interview based on the Structured Clinical Interview for DSM-III-R (SCID, Spitzer et al. 1987). The reliability of these two LTH diagnoses was poor $(\kappa=0 \cdot 34)$. Kendler et al. (1993) examined the generality of the covariates of stable diagnosis reported by Rice et al. (1992) by using a backward prediction paradigm that used the depressive features reported at time 2 to predict a diagnosis of MD was previously made at time 1 . This analytical approach, therefore, assumes that a diagnosis at time 2 identifies a positive case. The number of depressive symptoms again predicted a reliable diagnosis, as did impairment and help seeking associated with the worst episode, and the number of depressive episodes experienced over the lifetime. Reliably diagnosed MD was more heritable than MD assessed and diagnosed at only one occasion $\left(\mathrm{h}^{2}=0.70\right.$ for reliably diagnosed MD versus $h^{2}=0.49$ for time 1 (selfreport) $\mathrm{MD}$ and $\mathrm{h}^{2}=0.35$ for time 2 (SCID) MD), although Kendler et al. (1993) noted that the questionnaire and interview were not statistically equivalent indices of liability for MD.

On the basis of these findings both Rice and Kendler have argued for the adoption of a quantitative risk index, derived from the depressive features that predict reliable diagnosis, rather than a continued reliance on the more error-prone and less heritable RDC and DSM diagnoses. Two important issues remain to be addressed however.
First, it is necessary to demonstrate that unreliable diagnosis indexes only measurement error and non-genetic influences on liability to MD. A variety of transient or 'occasion-specific' influences on the recall of depressive episodes have been characterized, including internal symptom cues (current mood state) and external stress cues (recent salient life events). Aneshensel et al. (1987) reported that such effects are not random, but follow orderly patterns. The possibility that such effects may index genetic or other familial influences on true liability for MD has not, however, been formally tested in models that characterize the heritability of reliably diagnosed MD.

When only cross-sectional data are available, heritability $\left(\mathrm{h}^{2}\right)$ is calculated as the ratio of the estimated genetic variance $(\mathrm{Vg})$ to the total observed (phenotypic) variance $(\mathrm{Vp})$ - with the genetic variance estimated from the pattern of resemblance between different groups of relatives (Neale \& Cardon, 1992). Vp incorporates both genetic $(\mathrm{Vg})$ and environmental variance $(\mathrm{Ve})$, including that which is occasion-specific or 'unreliable' (Vk);

$$
\mathrm{h}^{2}=\mathrm{Vg} / \mathrm{Vp}, \quad \text { where } \mathrm{Vp}=\mathrm{Vg}+\mathrm{Ve}+\mathrm{Vk}
$$

In cross-sectional data, $\mathrm{Ve}$ and $\mathrm{Vk}$ are confounded and the estimated heritability therefore cannot exceed the reliability of the measured trait. If a trait is $100 \%$ heritable and measured with perfectly reliability then $\mathrm{Vg}=\mathrm{Vg}+\mathrm{Ve}+$ $\mathrm{Vk}=1.00$ (i.e. $\mathrm{Ve}=0$ and $\mathrm{Vk}=0$ ). If, however, the trait is $100 \%$ heritable but measurement of the trait is unreliable because of measurement error $(\mathrm{Ve}=0$ but $\mathrm{Vk}>0)$, then the genetic variance will be estimated as $<100 \%$ (i.e. $\mathrm{Vg}<\mathrm{Vg}+\mathrm{Ve}+\mathrm{Vk}=1)$. A method for estimating $\mathrm{Vk}$ using longitudinal data was developed by one of us (MCN), and this method was subsequently used to estimate the heritability of reliably diagnosed MD (Kendler et al. 1993). In that study, Vk was assumed to reflect errors of measurement and occasion-specific influences on the subject's recall of MD that are uncorrelated between relatives. Heritability of reliably diagnosed MD was calculated as

$$
\mathrm{h}^{2}=\mathrm{Vg} / \mathrm{Vp}, \quad \text { where } \mathrm{Vp}=\mathrm{Vg}+\mathrm{Ve}
$$

and Vk ('error') was estimated separately. As the denominator in (2) is smaller than the 
denominator in (1), by a factor equivalent to $\mathrm{Vk}$, $\mathrm{h}^{2}$ for reliably diagnosed MD is larger than $\mathrm{h}^{2}$ for MD assessed and diagnosed at only one occasion.

Kendler et al. (1993) did not formally test if Vk only indexes errors of measurement and occasion-specific influences on the subject's recall of MD that are uncorrelated between relatives. Multiple measurements that index both genetic and environmental influences, including measurement error, on occasion-specific variance have been reported previously, and the impact of such variance on the estimation of heritability discussed in detail elsewhere (Falconer, 1967). We therefore cannot assume $a$ priori that the estimated heritability for reliably diagnosed MD captures all the relevant genetic influences on liability to MD. It is possible that $\mathrm{Vk}$ is correlated between relatives, reflecting, for example, occasion-specific genetic influences on recent mood that are correlated with consistency of recall and true liability to MD.

The second issue raised by the findings reported by Rice et al. (1992) and Kendler et al. (1993) concerns the somewhat different correlates of reliably diagnosed MD reported by these two investigators. A variety of factors may account for the discrepant findings. First, each study assigned a positive case differently. Rice used the index diagnosis and a forward prediction paradigm to characterize stability over time, whereas Kendler used the follow-up diagnosis and a backward prediction paradigm. It is not clear, however, if a depressive history endorsed at index is equivalent in liability to one endorsed at follow-up given the lower re-test prevalence of negative affective states surveyed by rating scales (Jorm et al. 1989) and interviews (Helzer et al. 1981; Bromet et al. 1986, Eaton et al. 1989). Secondly, Kendler used different diagnostic criteria to assign a LTH of MD at time 1 and 2. At time 1, MD was diagnosed on the basis of a subset of self-rated DSM-III-R criteria. At time 2, MD was diagnosed using the full DSM-III-R criteria evaluated at personal interview. Thirdly, Rice used RDC criteria and Kendler used (variable) DSM-III-R criteria. Fourthly, the depressive features used to characterize stable diagnosis are likely to be highly correlated and the pattern of correlations may differ in selected and unselected samples. Fifthly, there may be differences in the correlates of consistent recall in community versus selected samples given the latter are likely to include a greater proportion of milder cases. Before the latter explanation can be assumed to account for all cross-study differences, and the data reported by Rice used as a basis for assigning different liability weight to subjects in both community and selected samples (Rice et al. 1992), points 1 to 4 require further consideration.

Lastly, temporal stability is just one test of validity (Rice et al. 1992). Robins \& Guze (1970) proposed five other criteria to establish diagnostic validity, including delimitation from other diagnoses. Does a reliable diagnosis of MD attenuate the association between MD and other disorders? If diagnostic reliability indexes severity of liability for MD (Rice et al. 1992; Kendler et al. 1993) and if co-morbidity between MD and GAD (Goethe et al. 1993; Brown et al. 1996) and between MD and panic disorder (Reich et al. 1993; Andrade et al. 1994; Pini et al. 1994) reflects a more severe depression, then reliably diagnosed MD may be associated with a higher risk for co-morbid anxiety disorder over the lifetime. If we are to understand the implications of reliable diagnoses we need to broaden our investigation to include a consideration of the multivariate pattern of risk associated with a reliable diagnosis.

Since the publication of Kendler et al. (1993), a LTH of DSM-III-R MD in this same sample of twins was surveyed again an average of 5 years after administration of the index interview. With these two wave interview data in hand, we are now able to address the following questions:

1 Do multiple surveys that cover the same risk period index occasion-specific genetic effects on liability to major depression?

2 Does the assignment of a positive case based on a diagnosis of MD at index versus follow-up affect the characterization of reliable diagnosis? Do the characteristics of an index diagnosis of MD differ from those reported at follow-up? What are the implications for the derivation of a quantitative risk index for depression?

3 How does diagnostic reliability affect the characterization of multivariate patterns of risk? What does this imply about the 
validity of reliably diagnosed MD and the boundary between MD and anxiety disorders?

\section{METHOD \\ Sample}

The sample of Caucasian female twins who are the subject of the present report are a subset of those registered with the population-based Virginia Twin Register (VTR). The VTR was formed from a systematic review of birth records in the Commonwealth of Virginia from 1915 onwards. Twins were initially mailed a selfreport questionnaire to which $64 \%$ of individuals responded. The true cooperation rate is likely to be higher than this figure suggests, however, because an unknown proportion of non-responding twins never received the questionnaire due to incorrect mailing addresses, incorrect forwarding of mail etc. Of the 2352 individual twins from 1176 twin pairs who returned the questionnaire, $2163(92 \%)$ twins from 1033 twin pairs were interviewed about their lifetime history of psychiatric disorder an average of $12 \cdot 3$ months (S.D. $=4 \cdot 0$ ) after receipt of the questionnaire (index lifetime history interview). Of these 2163 twins, 2002 (93\%) individuals twins from 938 twin pairs were interviewed over the telephone an average of 17 months later (S.D. $=3.7$ ) about onsets that occurred during the preceding year. Of these 2002 individuals, 1895 (95\%) individuals from 849 twin pairs completed another lifetime history interview and average of 44.3 months later (S.D. = 3.9) over the telephone (follow-up lifetime history interview). All interviews were conducted blind to the status of the co-twin by trained field staff who held a Master's degree in Social Work or had at least 2 years of clinical experience. Informed consent was obtained in writing prior to the personal interview, and verbally prior to the telephone interviews.

The data analysed here are for the 847 twin pairs who completed the depression module of both the index and follow-up lifetime history interviews: 496 monozygotic (MZ) and 351 dizygotic (DZ) twin pairs. Zygosity was determined by the twin's responses to standard questions regarding their physical similarity to their co-twin, the frequency with which they were confused as children, photographs and
DNA typing (Spence et al. 1988; Eaves et al. 1989). These 847 twin pairs were aged between 17 and 55 years at index $(\bar{x}=30$; S.D. $=7)$.

\section{Measures}

A LTH of MD was assessed at index and followup with an adapted version of the SCID (Spitzer et al. 1987) following DSM-III-R criteria (APA, 1987). At follow-up, only lifetime episodes reported to precede the index interview were analysed here in an effort to distinguish new onsets from unreliable recall. This may underestimate diagnostic reliability, however, because some subjects who recall previously denied symptoms at re-test may erroneously move the onset of these symptoms forward (Angst et al. 1984; Rubio-Stipec et al. 1992). Twenty subjects who reported a LTH of MD at both interviews reported an onset at follow-up that was later than their age at index, and these subjects are rated here as having a LTH of MD only at index. Characteristics of MD assessed at both interviews included the number of depressive symptoms, degree of reported impairment, help seeking, age at onset, length of worst episode and the number of lifetime episodes.

A LTH of GAD was assessed at index using Criterion D from DSM-III-R. Criterion A (unrealistic or excessive anxiety and worry (apprehensive expectation) about two or more life circumstances) and criterion $\mathrm{B}$ (the focus of the anxiety and worry in A is not attributable to another Axis 1 disorder) were not surveyed, and no diagnostic hierarchy or exclusion criteria were applied (criterion $\mathrm{C}$ and $\mathrm{E}$ ).

A LTH of panic disorder was assessed at index using criteria $\mathrm{A}(1), \mathrm{B}, \mathrm{C}$ and $\mathrm{D}$ from DSM-III-R. Criterion A(2) - panic attacks were not triggered by situations in which the person was the focus of others' attention - was not surveyed, and criterion $\mathrm{E}$-it cannot be established that an organic factor initiated and maintained the disturbance - was not applied. All diagnoses were assigned by computer algorithm.

\section{Twin analyses}

The twin model used here is based on a liabilitythreshold model that divides the variation in liability to MD into three classes: (i) additive genetic (A), which contribute twice as much to the correlation in MZ twins as DZ twins (because 
MZ twins share all their genes identical by descent, while DZ twins share on average only half their genes); (ii) family or 'common' environment (those familial factors which make twins similar in their liability to MD) (C), which contributes equally to the correlation in $\mathrm{MZ}$ and DZ twins; and (iii) individual specific environment (E), which reflects environmental experiences not shared by both members of a twin pair and therefore contribute to differences between them in their liability to MD. We have previously examined the equal environment assumption for MD (that the exposure to familial environmental risk factors for $M D$ is approximately equal in $\mathrm{MZ}$ and $\mathrm{DZ}$ twins) in this sample of twins and found no evidence to reject it (Kendler et al. 1994).

The twin model for MD utilizes both our index and follow-up diagnostic data. As pictured in Fig. $1 a$, the model assumes that there is a true latent liability to MD. Each of our two assessments of LTH are considered to be fallible indices of this true latent liability. The paths $\lambda_{1}$ and $\lambda_{2}$ represent the degree to which the assessments of LTH of MD obtained at the two time points reflect this true liability. The square of these paths is one potential measure of the reliability of these assessments. The other paths to LTH of MD at index and LTH of MD at follow-up ( $\mathrm{k}_{1}$ and $\mathrm{k}_{2}$, respectively) represent transient or 'occasion-specific' influences on the individual assessments of LTH of MD, including measurement error. By definition, $\lambda^{2}+\mathrm{k}^{2}=1 \cdot 0$. The latent liability to lifetime MD and the unreliable or 'occasion-specific' influences on recall/diagnosis at each measurement occasion are then modelled in a standard twin design, as outlined above, with the sources of variance in liability divided between additive genetic, common environmental and individual specific environmental factors.

It is important to emphasize two critical differences between this model and the standard twin model. First, this model estimates occasionspecific influences on recall that include errors of measurement (k). Secondly, it provides a direct estimate of the degree to which the individual assessments of LTH of MD index latent liability to MD $(\lambda)$. Lastly, this model differs from the model used by Kendler et al. (1993) in one important way. It has been extended to test whether occasion-specific influences on recall $(\kappa)$ are correlated between twins because of occasion-specific genetic $(\kappa a)$ or familial environmental influences $(\kappa \mathrm{c})$ on liability for MD.

\section{Characteristics of reliably diagnosed major depression (MD)}

The MD features at index that predict a diagnosis of MD at follow-up (forward prediction), and the MD features at follow-up that predict a diagnosis of MD at index (backward prediction) are characterized using bivariate and multiple logistic regression. The forward prediction sample comprises the 562 twins diagnosed with a LTH of MD at index, and the predictor variables are the number of symptoms, number of episodes, help seeking, impairment, worst episode duration and age at onset reported at index. This approach is comparable to that employed by Rice et al. (1992). The backward prediction sample comprises the 416 twins diagnosed with a LTH of MD at follow-up, and the predictor variables are the number of symptoms, the number of episodes, help-seeking, impairment, worst episode duration and age at onset reported at follow-up. This analysis is comparable to that employed by Kendler et al. (1993). To compare the depressive features of subjects diagnosed as having a LTH of MD only at index or only at follow-up $(N=406)$, bivariate and multiple logistic regression is used to model the depressive features that predicted the diagnosis was assigned only at index (versus followup).

\section{Reliable diagnosis and co-morbidity}

To determine if co-morbidity significantly influences the odds of a reliable diagnosis for a LTH of MD, the logistic regression continuation ratio test (CRT, MacClean, 1988) was used to compare the odds that MD was diagnosed once or twice (CRT 1), versus twice (CRT 2), given a lifetime history of GAD or panic disorder at index. If the slope of the regression line is constant across CRT 1 and CRT 2, then the continuation ratio test statistic (D) will be insignificantly different from 0 . If $D$ is not insignificantly different from zero this indicates that the odds that MD is reliably diagnosed increase linearly if the subject has a LTH of GAD or panic disorder at index. If $D$ is significantly greater than 0 then this indicates that the odds of a reliable diagnosis increase in 


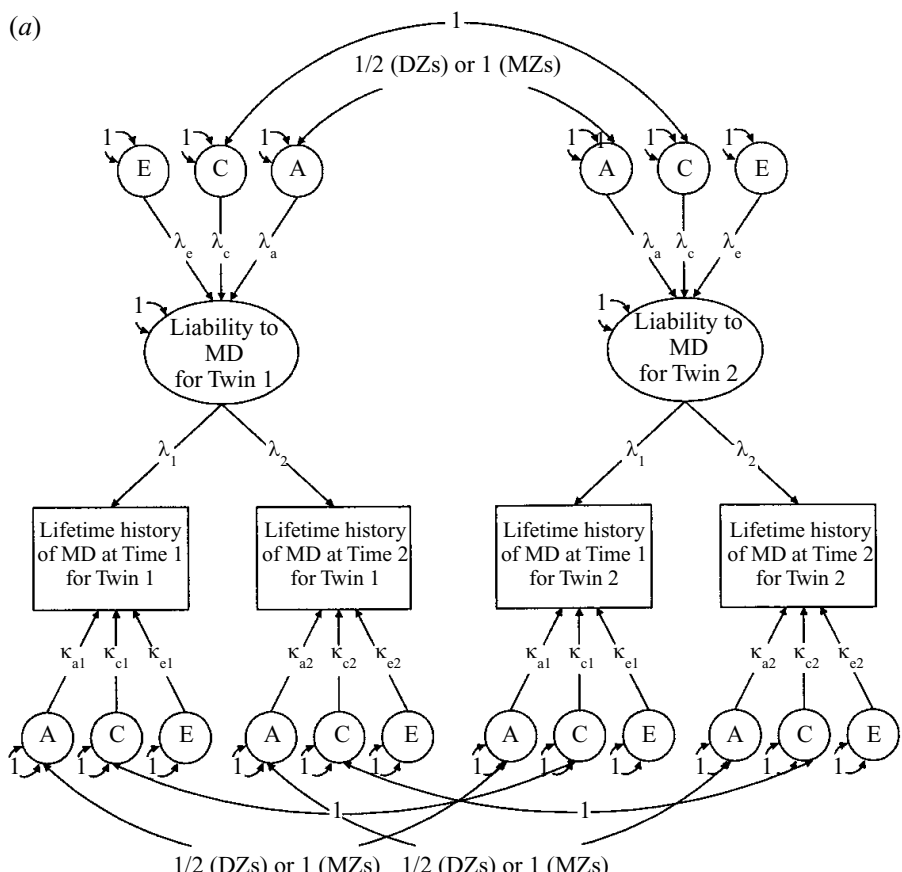

(b)

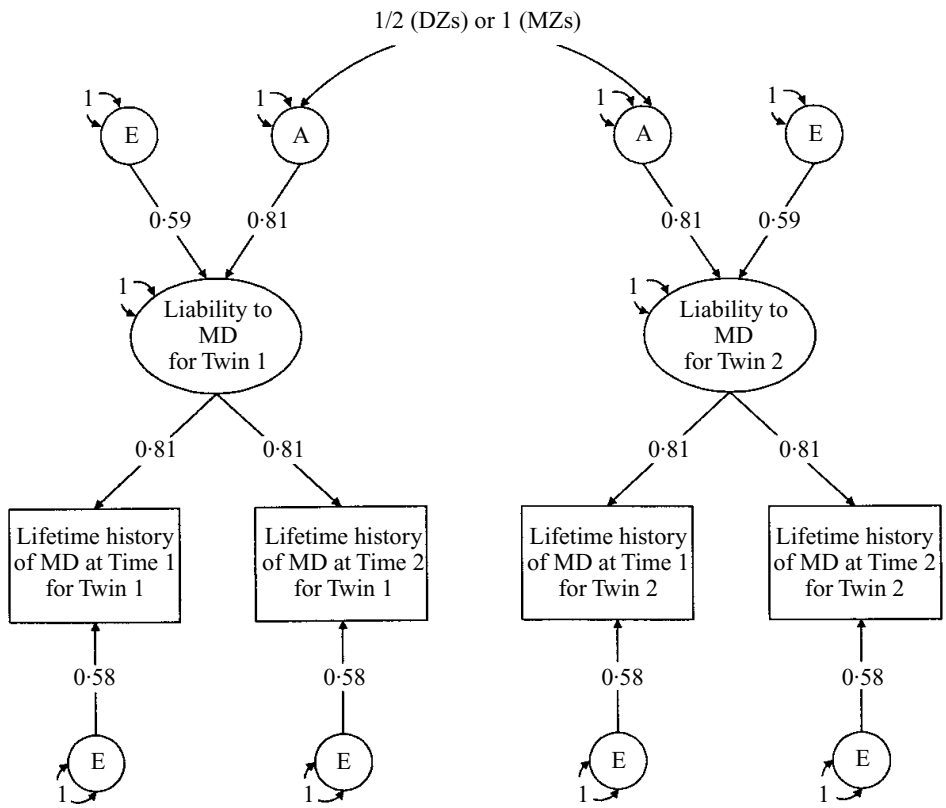

Fig. 1. (a) A twin model for the heritability of liability to a lifetime history (LTH) of major depression (MD) including transient influences on the recall and diagnosis of a LTH of MD. This model assumes that there is a true (latent) liability to a LTH of MD, which is indexed by two assessments, at time 1 and 2. The paths $\lambda_{1}$ and $\lambda_{2}$ represent the degree to which these assessments reflect true liability to LTH of MD. The square of these paths is a measure of the reliability of these assessments. The other paths to a LTH of MD $\left(\kappa_{\mathrm{a}}, \kappa_{\mathrm{e}}\right.$ and $\left.\kappa_{\mathrm{e}}\right)$ represent transient influences on each assessment of a LTH of MD, which may reflect genetic effects on the recall and diagnosis of MD at time $1\left(\kappa_{\mathrm{a} 1}\right)$ or time $2\left(\kappa_{\mathrm{a} 2}\right)$, shared or common environmental influences on the recall and diagnosis of MD at time $1\left(\kappa_{\mathrm{c} 1}\right)$ or time $2\left(\kappa_{\mathrm{c} 2}\right)$, or unshared or individual-specific environmental influences and/or measurement error on the 
a non-linear fashion when the subject has a LTH of GAD or panic disorder at index, and if $\mathrm{D}$ is significantly less than 0 then this indicates that the odds of a reliable diagnosis of MD decrease when the subject has a LTH of GAD or panic disorder at index.

\section{Software}

The twin modelling is performed using $\mathrm{Mx}$ (Neale, 1997) with the best-fitting model in our analyses selected using Akaike's Information Criterion (AIC) (Akaike, 1987; Williams \& Holahan, 1994). Logistic regression is performed using forward selection, using a $P<0.05$ significance level to enter variables in the model (SAS Institute Inc., 1990). The continuation ratio test is calculated using a SAS program written by Dr Charles Gardner.

\section{RESULTS}

\section{Prevalence and reliability of a lifetime history of major depression}

The index and follow-up LTH interviews were conducted an average of 62.6 months apart (S.D. $=4 \cdot 9$, range $=46-92)$. The retest interval is not significantly different for $\mathrm{MZ}$ and $\mathrm{DZ}$ twin pairs (Wilcoxin two-sample test, $Z=0.67$, $P=0.50)$. At index, the prevalence of a LTH of $\mathrm{MD}$ is $33.2 \%(562 / 1694)$. At follow-up, the

Table 1. Temporal stability over 5 years for a diagnosis of a lifetime history of major depression

\begin{tabular}{llr}
\hline \hline & \multicolumn{1}{c}{$\begin{array}{c}\text { Lifetime history of } \\
\text { major depression at follow-up }\end{array}$} \\
\cline { 2 - 3 } $\begin{array}{l}\text { Lifetime history of } \\
\text { major depression at index }\end{array}$ & Present & Absent \\
\hline $\begin{array}{l}\text { Present } \\
\text { Absent }\end{array}$ & $286(\mathrm{~A})$ & $276(\mathrm{~B})$ \\
\hline \hline
\end{tabular}

prevalence of MD for the lifetime preceding the index interview is $24.6 \%(416 / 1694)$. This difference is statistically significant (McNemar Test, $\left.\chi^{2}=52.5 \mathrm{df}=1, P<0.001\right)$.

If a diagnosis of MD at index is defined as the criterion variable, and a diagnosis of MD at follow-up is defined as the 'test' variable, then the sensitivity of our diagnoses is $51 \%(\mathrm{~A} / \mathrm{A}+\mathrm{B}$ in Table 1). The specificity is $89 \%(\mathrm{D} / \mathrm{C}+\mathrm{D})$, the positive predictive value is $69 \%(\mathrm{~A} / \mathrm{A}+\mathrm{C})$, and the negative predictive value is $78 \%$ (D/B + D). Kappa (Cohen, 1960) for LTH of MD is 0.43 (95\% confidence interval $0 \cdot 38-0.48$ ), indicating only fair agreement across time.

\section{Twin models}

The first twin model, Model 1 (Fig. $1 a$, the 'full model'), fit the data very well ( $\chi^{2}=1 \cdot 95, \mathrm{df}=6$, $P=0.92$, AIC $=-10.05)$. Five paths in this model were estimated at zero: familial (shared) environmental influences on liability to reliably diagnosed MD $(\lambda c)$, and the occasion-specific genetic and familial environmental influences on a MD diagnosis at index ( $\kappa \mathrm{a} 1$ and $\kappa \mathrm{c} 1)$ and follow-up ( $\kappa \mathrm{a} 2$ and $\kappa \mathrm{c} 2$ ). These parameters could, therefore, be set to zero in Model 2 with no change in model fit $\left(\chi^{2}=1.95, \mathrm{df}=11, P=\right.$ $0 \cdot 99$, AIC $=-20 \cdot 05)$. Model 2 therefore suggests that familial environmental factors and occasion-specific genetic effects do not influence liability to MD. To test if liability to reliably diagnosed MD can be attributed exclusively to genetic effects $(\lambda \mathrm{a})$, the environmental liability path $(\lambda$ e) was set to zero in Model 3. Compared to Model 2, Model 3 provided a significantly worse fit to the data $\left(\chi^{2}=27 \cdot 22, \mathrm{df}=12, P<\right.$ $0 \cdot 01, \mathrm{AIC}=3 \cdot 22$ ) suggesting that experiences that are not shared by twins significantly contribute to their liability for MD. We next tested if liability to MD can be attributed exclusively to such experiences and therefore set

recall and diagnosis of MD at time $1\left(\kappa_{\mathrm{e} 1}\right)$ or time $2\left(\kappa_{\mathrm{e} 2}\right)$. The model is constrained such that $\lambda^{2}+(\kappa \mathrm{a}+\kappa \mathrm{c}+\kappa \mathrm{e})^{2}=1$. Both the true liability for, and the transient influences on the recall and diagnosis of, a LTH of MD are modelled in a standard twin design with the sources of variance in each divided between additive genetic (A), common (C) environmental, and individual-specific (E) environmental factors. By definition, the 'common' environmental components are perfectly correlated in all twins, while the 'individual-specific' environment is uncorrelated. Additive genetic factors are perfectly correlated in monozygotic (MZ) twins and correlated 0.50 in dizygotic (DZ) twins. Lower case letters (a, c, e) are used to label the paths from these factors. The individual paths represent standardized regression coefficients, so that the proportion of variance in the dependent variables accounted for by the independent variables is equal to the square of the connecting path. Heritability of reliably recalled and diagnosed LTH of MD for example equals $\lambda \mathrm{a}^{2}$. Observed variables are depicted in boxes and latent variables in circles and ellipses. (b) Parameter estimates for the best fitting model (model 5). Parameter estimates are constrained to be equal for twin 1 and twin 2. No evidence was found for common environmental influences on liability to a LTH of MD. No evidence was found for transient genetic or common environmental influences on the recall and diagnosis of a LTH of MD. Heritability of true liability to a $\mathrm{LTH}$ of $\mathrm{MD}=0 \cdot 81^{2}=66 \%$. 
Table 2. Prediction of reliable reporting of lifetime history of major depression:

I. Bivariate analyses

\begin{tabular}{|c|c|c|c|c|c|c|c|c|}
\hline \multirow[b]{3}{*}{ Predictors } & \multicolumn{8}{|c|}{ Bivariate predictors of reliable recall of LTH of MD } \\
\hline & \multicolumn{4}{|c|}{$\begin{array}{l}\text { I. Forward prediction } \\
\text { Characteristics reported at index that predict a } \\
\text { LTH of MD is recalled at follow-up }\end{array}$} & \multicolumn{4}{|c|}{$\begin{array}{l}\text { II. Backward prediction } \\
\text { Characteristics reported at follow-up that predict a } \\
\text { LTH of MD was previously recalled at index }\end{array}$} \\
\hline & $\beta$ & $\chi^{2}$ & $P<$ & OR $(95 \% \mathrm{CI})$ & $\beta$ & $x^{2}$ & $P<$ & OR $(95 \% \mathrm{CI})$ \\
\hline No. of symptoms & $0 \cdot 41$ & $31 \cdot 62$ & $0 \cdot 01$ & $1 \cdot 50(1 \cdot 30,1 \cdot 73)$ & $0 \cdot 31$ & $11 \cdot 74$ & $0 \cdot 01$ & $1 \cdot 37(1 \cdot 14,1 \cdot 64)$ \\
\hline Help-seeking & $1 \cdot 06$ & $26 \cdot 31$ & $0 \cdot 01$ & $2.88(1.92,4.31)$ & $0 \cdot 34$ & $2 \cdot 60$ & $0 \cdot 11$ & $1 \cdot 41(0 \cdot 93,2 \cdot 15)$ \\
\hline No. of episodes & $0 \cdot 14$ & $1 \cdot 84$ & $0 \cdot 18$ & $1.15(0.94,1.34)$ & $0 \cdot 31$ & $6 \cdot 37$ & $0 \cdot 01$ & $1.36(1.07,1.73)$ \\
\hline Impairment & $0 \cdot 39$ & $10 \cdot 44$ & $0 \cdot 01$ & $1 \cdot 47(1 \cdot 16,1 \cdot 85)$ & $0 \cdot 20$ & $2 \cdot 33$ & $0 \cdot 13$ & $1.22(0.94,1.59)$ \\
\hline Duration & $0 \cdot 07$ & $0 \cdot 71$ & $0 \cdot 40$ & $1.07(0.91,1.26)$ & $0 \cdot 34$ & $9 \cdot 26$ & $0 \cdot 01$ & $1.41(1.13,1.73)$ \\
\hline Age at onset & $-0 \cdot 01$ & $0 \cdot 08$ & $0 \cdot 77$ & $0.99(0.97,1.02)$ & $-0 \cdot 01$ & $0 \cdot 06$ & $0 \cdot 81$ & $0.99(0.97,1.02)$ \\
\hline
\end{tabular}

Table 3. Prediction of reliable reporting of lifetime history of major depression: do the same characteristics predict forward and backward discordance? II. Multivariate analyses

\begin{tabular}{|c|c|c|c|c|c|c|c|c|}
\hline \multirow[b]{3}{*}{ Predictors } & \multicolumn{8}{|c|}{ Multivariate predictors of reliable recall of LTH of MD } \\
\hline & \multicolumn{4}{|c|}{$\begin{array}{l}\text { I. Forward prediction } \\
\text { Characteristics reported at index that predict a } \\
\text { LTH of MD is recalled at follow-up }\end{array}$} & \multicolumn{4}{|c|}{$\begin{array}{l}\text { II. Backward prediction } \\
\text { Characteristics reported at follow-up that predict a } \\
\text { LTH of MD was previously recalled at index }\end{array}$} \\
\hline & $\beta$ & $x^{2}$ & $P<$ & OR $(95 \% \mathrm{CI})$ & $\beta$ & $x^{2}$ & $P<$ & OR $(95 \% \mathrm{CI})$ \\
\hline Help-seeking & $0 \cdot 89$ & $17 \cdot 13$ & $0 \cdot 01$ & $2 \cdot 44(1 \cdot 60,3 \cdot 72)$ & * & - & - & - \\
\hline No. of symptoms & $0 \cdot 38$ & $19 \cdot 35$ & $0 \cdot 01$ & $1.46(1.23,1.73)$ & $0 \cdot 26$ & $7 \cdot 92$ & $0 \cdot 01$ & $1 \cdot 30(1.08,1 \cdot 57)$ \\
\hline No. of episodes & $*$ & - & - & - & $0 \cdot 30$ & $5 \cdot 77$ & 0.02 & $1.35(1.06,1.73)$ \\
\hline Impairment & $*$ & - & - & - & $*$ & - & - & - \\
\hline Duration & $*$ & - & - & - & $0 \cdot 30$ & $6 \cdot 82$ & $0 \cdot 01$ & $1 \cdot 35(1.08,1 \cdot 70)$ \\
\hline Age at onset & $*$ & - & - & - & $*$ & - & - & - \\
\hline
\end{tabular}

* Variable excluded in multiple logistic regression using forward selection.

the genetic liability path to zero $(\lambda \mathrm{a}=0)$ in Model 4. This led to an even worse model fit $\left(\chi^{2}=99 \cdot 18, \quad \mathrm{df}=12, \quad P<0 \cdot 01, \quad \mathrm{AIC}=75 \cdot 18\right)$ indicating that both genetic and non-familial environmental risk factors significantly contribute to liability for MD. The final model fit to these data (Model 5) equated the reliability of our index and follow-up interviews $\left(\lambda_{1}=\lambda_{2}\right)$, but was in all other respects identical to Model 2. In model 5 , these two reliability paths could be equated with no significant reduction in model fit $\left(\chi^{2}=2 \cdot 51, \mathrm{df}=12, P=0 \cdot 998\right.$, AIC $=$ $-21 \cdot 48$ ) which suggests that the index and follow-up diagnoses are not significantly different indices of liability for MD. Model 5 provides the best fit to these longitudinal diagnostic data (Fig. $1 b$ ), estimating that liability to MD in this unselected sample of twins reflects both genetic $\left(\lambda \mathrm{a}^{2}=66 \%, \mathrm{CI}=53 \%, 78 \%\right)$ and environmental risk factors unshared by co-twins $\left(\lambda \mathrm{e}^{2}=34 \%, \quad \mathrm{CI}=22 \%, \quad 47 \%\right)$. Occasion- specific influences on the recall and diagnosis of MD at onset and follow-up reflect environmental effects unshared by relatives and/or measurement error, and together these account for $34 \%$ $(\mathrm{CI}=22 \%, 47 \%)$ of the total variance in the diagnosis of LTH of MD at each interview. The estimated heritability of a reliably diagnosed LTH of MD is calculated as $\mathrm{Vg} / \mathrm{Vp}=0.66$ where $\mathrm{Vg}=\lambda \mathrm{a}^{2}$ and $\mathrm{Vp}=\lambda \mathrm{a}^{2}+\lambda \mathrm{e}^{2}$. The estimated heritability of LTH of MD assessed and diagnosed on the basis of a single psychiatric interview is calculated as $\mathrm{Vg} / \mathrm{Vp}=0.43$ where $\mathrm{Vg}=\lambda \mathrm{a}^{2}$ and $\mathrm{Vp}=\lambda \mathrm{a}^{2}+\lambda \mathrm{e}^{2}+\kappa \mathrm{e}^{2}$.

\section{Characteristics of reliably diagnosed major depression}

Forward prediction: what depressive features at time 1 predict a second lifetime diagnosis of major depression at time 2?

Using a diagnosis of MD at index to designate a positive case, the bivariate predictors of a reliable 
Table 4. Prediction of the temporal order of inconsistent recall of a LTH of MD: what characteristics distinguish subjects who recall a LTH of MD only at index from those who report a LTH of MD only at follow-up?

Characteristics of inconsistent recall of a LTH of MD

\begin{tabular}{|c|c|c|c|c|c|c|c|c|c|c|}
\hline \multirow[b]{3}{*}{ Predictors } & \multicolumn{10}{|c|}{ Characteristics of inconsistent recall of a LTH of MD } \\
\hline & \multicolumn{5}{|c|}{$\begin{array}{c}\text { I. Bivariate regression } \\
\text { Characteristics that predict an inconsistently recalled } \\
\text { LTH of MD will be recalled only at index, and not at } \\
\text { follow-up }\end{array}$} & \multicolumn{5}{|c|}{$\begin{array}{c}\text { II. Multivariate regression } \\
\text { Characteristics that predict an inconsistently recalled } \\
\text { LTH of MD will be recalled only at index, and not at } \\
\text { follow-up }\end{array}$} \\
\hline & $\beta$ & $x^{2}$ & S.E. & $P<$ & OR $(95 \%$ CI $)$ & $\beta$ & $x^{2}$ & S.E. & $P<$ & OR $(95 \% \mathrm{CI})$ \\
\hline Help-seeking & $-0 \cdot 84$ & $12 \cdot 42$ & $0 \cdot 24$ & $0 \cdot 01$ & $0 \cdot 43(0 \cdot 27,0 \cdot 69)$ & -0.93 & 13.94 & $0 \cdot 25$ & $0 \cdot 01$ & $0 \cdot 39(0 \cdot 24,0 \cdot 64)$ \\
\hline No. of symptoms & $0 \cdot 27$ & $7 \cdot 42$ & $0 \cdot 10$ & $0 \cdot 01$ & $1 \cdot 31(1.08,1 \cdot 59)$ & $0 \cdot 35$ & $10 \cdot 63$ & $0 \cdot 11$ & $0 \cdot 01$ & $1.42(1 \cdot 15,1 \cdot 75)$ \\
\hline No. of episodes & $0 \cdot 03$ & $0 \cdot 06$ & $0 \cdot 12$ & $0 \cdot 81$ & $1 \cdot 03(0 \cdot 81,1 \cdot 31)$ & $*$ & - & - & - & - \\
\hline Impairment & $0 \cdot 13$ & $0 \cdot 84$ & $0 \cdot 15$ & $0 \cdot 36$ & $1 \cdot 14(0.86,1 \cdot 52)$ & $*$ & - & - & - & - \\
\hline Duration & $-0 \cdot 22$ & 3.98 & $0 \cdot 11$ & 0.05 & $0.81(0.65,0.97)$ & $*$ & - & - & - & - \\
\hline Age at onset & $-0 \cdot 02$ & $2 \cdot 86$ & $0 \cdot 01$ & $0 \cdot 09$ & $0.98(0.95,1.01)$ & $-0 \cdot 04$ & $6 \cdot 04$ & $0 \cdot 01$ & $0 \cdot 01$ & $0.97(0.94,0.99)$ \\
\hline
\end{tabular}

* Variable excluded in multiple logistic regression.

Table 5. Does co-morbidity affected the odds that major depression will be reliably diagnosed?

\begin{tabular}{|c|c|c|c|c|c|c|c|c|c|c|}
\hline \multirow[b]{2}{*}{ Risk factor at index } & \multicolumn{5}{|c|}{ LTH of MD recalled once or twice $v$. never } & \multicolumn{5}{|c|}{ LTH of MD recalled twice $v$. once } \\
\hline & $\beta$ & S.E. & $x^{2}$ & $P<$ & Odds ratio $(\mathrm{CI})$ & $\beta$ & S.E. & $x^{2}$ & $P<$ & Odds ratio $(\mathrm{CI})$ \\
\hline LTH of GAD & $2 \cdot 23$ & $0 \cdot 28$ & $65 \cdot 49$ & $0 \cdot 0001$ & $9 \cdot 33(5 \cdot 43,16 \cdot 03)$ & $2 \cdot 39$ & $0 \cdot 21$ & $125 \cdot 43$ & $0 \cdot 0001$ & $10 \cdot 95(7 \cdot 20,16 \cdot 65)$ \\
\hline LTH of panic disorder & $1 \cdot 61$ & $0 \cdot 28$ & $32 \cdot 57$ & $0 \cdot 0001$ & $5 \cdot 00(2 \cdot 88,8 \cdot 70)$ & $0 \cdot 91$ & $0 \cdot 29$ & $9 \cdot 81$ & $0 \cdot 002$ & $2 \cdot 47(1.40,4 \cdot 36)$ \\
\hline
\end{tabular}

LTH, lifetime history; GAD, generalized anxiety disorder; MD, major depression.

diagnosis are help seeking $(\mathrm{OR}=2 \cdot 9)$, an increasing number of symptoms $(\mathrm{OR}=1 \cdot 5)$ and an increasing level of impairment $(\mathrm{OR}=1 \cdot 5$, Table 2). The multivariate predictors are help seeking $(\mathrm{OR}=2 \cdot 4)$ and an increasing number of symptoms $(\mathrm{OR}=1 \cdot 5$, Table 3$)$.

Backward prediction: what depressive features at time 2 predict a previous diagnosis of $M D$ at time 1 ?

Using a diagnosis of MD at follow-up to designate a positive case, the bivariate predictors of a reliable diagnosis are help seeking (OR = $1 \cdot 4)$, duration of worst episode $(\mathrm{OR}=1 \cdot 4)$, and an increasing number of symptoms $(\mathrm{OR}=1 \cdot 4$, Table 2). The multivariate predictors are duration of worst episode $(\mathrm{OR}=1 \cdot 3)$, the number of lifetime episodes $(\mathrm{OR}=1 \cdot 3)$ and an increasing number of symptoms $(\mathrm{OR}=1 \cdot 3$, Table 3$)$. Although the predictors of reliability differ depending on how we designate a positive case, inspection of the $95 \%$ confidence intervals for the estimated odds ratios indicates that the prediction of reliability does not differ significantly across paradigms.
The temporal order of unreliable diagnosis: what distinguishes a diagnosis given only at index from one given only at follow-up?

Compared with a diagnosis of LTH of MD that is made only at follow-up, a diagnosis of LTH of MD that is made only at index is characterized by an increasing number of symptoms $(\mathrm{OR}=$ $1 \cdot 31)$, less help-seeking $(\mathrm{OR}=0 \cdot 4)$, and a shorter duration of illness $(\mathrm{OR}=0.8)$ in bivariate regressions (Table 4). Using multiple regression, a diagnosis that is made only at index is characterized by an increasing number of symptoms $(\mathrm{OR}=1 \cdot 4)$, less help-seeking $(\mathrm{OR}=$ $0 \cdot 4)$ and a slightly younger age at onset $(\mathrm{OR}=$ $0 \cdot 97$, Table 4).

\section{Co-morbidity associated with a reliably diagnoses lifetime history of major depression}

The lifetime prevalence of GAD at index is $6.3 \% \quad(N=107 / 1694)$. GAD is significantly associated with MD in this sample $(\mathrm{OR}=9 \cdot 3$, Table 5), and the odds of a reliable diagnosis of MD increase when the subject has a history of GAD diagnosed at index $(\mathrm{D}=0 \cdot 54$, variance 
$\mathrm{D}=0 \cdot 10, Z$ test $=1 \cdot 71, P=0 \cdot 08$, Table 5). The prevalence of GAD among subjects diagnosed with a LTH of MD at both index and follow-up, only at index, only at follow-up, or at neither index or follow-up is $23 \cdot 8 \%(N=68 / 286), 7 \cdot 7 \%$ $(N=20 / 276), 2 \cdot 3 \% \quad(N=3 / 130)$ and $1.6 \%$ $(N=16 / 1002)$ respectively.

The lifetime prevalence of panic disorder at index is $2.2 \%(N=38 / 1694)$. Panic disorder is significantly associated with MD in this sample $(\mathrm{OR}=5 \cdot 0$, Table 5$)$ and the odds of a reliable diagnosis of MD increase when a history of panic disorder is diagnosed at index $(\mathrm{D}=-0 \cdot 43$, variance $\mathrm{D}=0 \cdot 14, \quad Z$ test $=1 \cdot 16, \quad P=0 \cdot 25$, Table 5). The prevalence of panic disorder among subjects diagnosed with a LTH of MD at both index and follow-up, only at index, only at follow-up, or at neither index or follow-up is $11.9 \%(34 / 286), 5 \cdot 4 \%(15 \cdot 276), 4.6 \%(6 / 130)$ and $1.7 \%(17 / 1002)$ respectively.

\section{DISCUSSION}

An unreliable diagnosis of LTH of MD in this epidemiological sample of adult female twins indexes only measurement error and/or transient non-familial influences on liability to MD. We found no evidence for genetic influences on transient or 'occasion-specific' effects on our index and follow-up assessments of LTH of MD. This finding provides further support for the utility of a quantitative risk index derived from the features of reliably recalled and diagnosed depressive histories (Rice et al. 1992; Kendler et al. 1993). A reliably diagnosed LTH of MD in this sample of unselected twins is $50 \%$ more heritable than a LTH of MD surveyed and diagnosed at only one occasion $\left(\mathrm{h}^{2}=0.66\right.$ versus $\mathrm{h}^{2}=0.43$ respectively). This underscores the impact of measurement error and other occasion-specific influences on the recall and rating of $\mathrm{MD}$, and the higher mean genetic liability of subjects who consistently report a LTH of MD in epidemiological surveys. Liability to reliably diagnosed MD also reflects the cumulative impact of experiences unshared by relatives, compatible with other aetiological models of MD (Brown \& Harris, 1978; Bowlby, 1980).

These results confirm those previously reported by Kendler et al. (1993a), using a selfreport measure of MD and a follow-up SCID- based interview, and accord well with the recent findings of McGuffin et al. (1996). McGuffin and colleagues estimated the heritability of DSM-IV major depression in 177 pairs of twins ascertained from the Maudsley Hospital Twin Register. These twins were administered a PSEbased personal interview if their first contact diagnosis met DSM-III criteria for affective disorder. Diagnosis of DSM-IV unipolar depression, therefore, reflects diagnostic agreement for a LTH of MD (albeit variably defined) over two occasions, which is broadly compatible with our concept of reliably diagnosed MD. Assuming a population risk of $16.6 \%$ in women, the heritability of MD in the Maudsley twins is estimated at $75 \%$. The prevalence of a reliably recalled LTH of MD in the VTR twins is $16.9 \%$, and heritability is estimated here at $66 \%(95 \%$ CI 53-78\%). The comparability of these findings is important because the Maudsley sample represents the largest systematically ascertained clinical sample of twins with unipolar depression.

The time 1 depressive features that predicted a LTH of MD was diagnosed again at time 2 are an increasing number of symptoms and helpseeking. These findings accord with those previously reported by Rice et al. (1992) as helpseeking in the VTR twins subsumes treatment and hospitalization as a result of seeking the help of a medical professional. This suggests that the features of a reliably diagnosed LTH of MD do not vary for DSM-III-R and RDC definitions of depression, and that the correlates of reliable recall do not differ in community and selected samples. If a diagnosis of LTH of MD at follow-up is used to designate a positive case, the depressive features that predict a LTH of MD was previously diagnosed at index are an increasing number of symptoms, an increasing number of lifetime episodes and a longer (worst) episode of illness. These findings partly replicate those reported by Kendler et al. (1993). In that study episode duration was not a significant predictor of the questionnaire-based diagnosis, and help-seeking and impairment were more strongly (and significantly) associated with a reliable diagnosis. The differences between the present study and that conducted by Kendler et al. (1993) are likely to reflect the impact of criterion variance in Kendler's study, whereas the differences between the findings reported by Kendler et al. (1993) and Rice et al. (1992) are 
likely to reflect both criterion variance in Kendler et al. (1993) and differences between subjects diagnosed with a LTH of MD only at index or only at follow-up. In the present study, the latter report relatively fewer symptoms, a longer (worst) episode of illness and more help seeking. It is of interest that McGuffin et al. (1996) reported a significantly higher $\mathrm{MZ}$ to $\mathrm{DZ}$ concordance ratio associated with depressive episodes of $<13$ months, which they suggest may reflect a greater genetic loading for depressions of shorter duration. Furthermore, Kendler \& Gardner (1998) report that an increasing number of MD symptoms predict an increased risk for future depressive episodes and a heightened co-twin risk for MD. Taken together, these data suggest that a reliably diagnosed LTH of MD indexes the memorability of depression, help-seeking and severity of liability, with a LTH diagnosed only at followup likely to index a slightly lower mean liability to MD than a LTH recalled only at index. Quantitative caseness indices of MD may, therefore, need to incorporate the temporal sequence of LTH diagnoses to accurately characterize weighted risk estimates in longitudinal surveys.

McGuffin et al. (1996) have emphasized that estimates of heritability depend, in part, on the (estimated) population frequency of MD, but suggest that differences in prevalence across surveys (as a function of variable threshold placement) are unlikely to affect the overall pattern of results. Although this may be true for univariate model fitting results, the positioning of the diagnostic (or reliability) threshold may have important implications for the characterization of multivariate patterns of risk.

In the VTR twins, a history of GAD is strongly associated with a reliably diagnosed LTH of MD. The genetic correlation between GAD and MD diagnosed on the basis of a single interview has been estimated at unity (Kendler et al. 1992 b; Roy et al. 1995), suggesting that a common set of genes underlie the familial component of risk for both disorders. The present findings further suggest that a history of chronic ( $>6$ month) GAD indexes a higher mean liability for $\mathrm{MD}$, consistent with the finding that co-morbidity between $\mathrm{MD}$ and GAD is associated with a greater severity of depression in clinical samples (Goethe et al.
1993; Brown et al. 1996). A history of panic disorder also predicts reliably diagnosed MD, supporting the suggestion that co-morbidity reflects a more severe illness (Reich et al. 1993; Andrade et al. 1994; Pini et al. 1994). In the analyses reported here, we have assumed that risk for MD reflects a normally distributed multifactorial liability and that a reliable diagnosis of MD indexes a higher mean liability. Although we consider it very unlikely that a higher liability to MD would not subsume the risk factors for a lower liability to MD (a cumulative risk model), this does not preclude the possibility that there are risk factors that operate only at higher liability levels. For example, if co-morbidity reflects an epiphenomena of illness severity then co-morbidity will characterize more severe depressions.

A history of GAD or panic disorder increases the odds that MD will be reliably diagnosed over a 5-year period. This suggests, first, that more reliable diagnostic assignments may not serve to validate the existing diagnostic boundaries that have been drawn between disorders and, second, that models which formally test if co-morbidity reflects epiphenomena associated with severity of liability of the focal disorder should be more widely utilized (Neale \& Kendler, 1995).

The present data also highlight the modest 4 to 8 year reliability $(\kappa=0.43)$ of a SCID-based LTH of MD in this population based sample. This is, however, very similar to the 1 day to 2 week reliability of the SCID in 202 non-patients ascertained via community advertisements $(\kappa=$ 0.49) (Williams et al. 1992) and the 18 month reliability of RDC MD in an epidemiological sample of 391 women $(\kappa=0.41)$ (Bromet et al. 1986). Kappa ranges between $0 \cdot 21$ and 0.75 for a LTH of MD in other unselected samples retested after 5 days to 7 years using a variety of different interviews and diagnostic criteria (Prusoff et al. 1988).

Cannell \& Fowler (1963) suggested that follow-up interviews may provide progressively less accurate information due to lowered subject motivation over time. In the present study, the (personal) index and (telephone) follow-up interviews are statistically equivalent indices of liability to MD. The significantly lower lifetime prevalence of MD estimated at follow-up here may reflect a 're-test artefact', an explanation 
previously invoked to account for the lower retest prevalence of (negative) affective states surveyed by rating scales (Jorm et al. 1989) or at interview (Helzer et al.1981; Bromet et al. 1986; Eaton et al. 1989). It is also possible that the different memory tasks required at each occasion may be partly responsible for the difference in lifetime prevalence estimated by each interview. At index, subjects are asked to recall any previous episode of MD. At follow-up, subjects are asked to recall any previous MD episode that occurred prior to the index interview. Given that only 20 subjects who reported a history of MD at onset reported that their histories of MD post-dated the onset interview at follow-up, this explanation is unlikely, however, to account for all the variance in prevalence across time.

Wells et al. (1988) concluded that disagreement in LTH assignments over time reflected unreliability and a re-test artefact that affected the reporting of certain depressive symptoms, rather than the method of administration. Weeks et al. (1983) found that subjects interviewed over the telephone were less likely than subjects interviewed face-to-face to report conditions for which they had been hospitalized, but, when they did report such conditions, they did so more accurately. These findings suggests that a substantial amount of time (Sobin et al. 1993) and money (Weeks et al. 1983) could be saved by assessing MD over the telephone, although longitudinal data are likely to incorporate a retest artefact associated with lower endorsement rates of negative affective states.

\section{Limitations}

The results presented here should be interpreted in light of the following limitations. First, reliability is assessed by kappa, and modelled using dichotomous diagnostic assignments, which may exaggerate the cross-time disagreement in the reporting of MD symptoms/ duration (Wainwright et al. 1997). Secondly, our twin model of diagnostic reliability assumes that error is random across subjects and is not informative regarding the type of misclassification (Carey \& Gottesman, 1978). Thirdly, the sample comprises only women. Although Rice et al. (1992) reported no impact of gender on the stability of MD diagnoses in relatives of patients, Angst et al. (1984) reported that men forgot certain symptoms of depression more often than women did and Wilhelm \& Parker (1994) found that women were more likely than men to 'remember' episodes of depression that had not previously reached case criteria. Fourthly, given the sex differences in prevalence and familial transmission of MD (Rice et al. 1984; Weissman et al. 1991; Wilhelm et al. 1997), the present findings may not be replicated with a male sample. Finally, the findings derived from latent variable models and logistic regression are informative in so far as the model assumptions are supportable or their violation has a negligible effect upon the parameter estimates obtained. For example, no significant common familial environmental influences on liability were identified here although separation from parents, due to factors such as divorce or parental illness prior to age 17, has been previously shown to have a small $(1.6 \%)$ but significant effect on risk of MD when separation is modelled as a specified index of the common familial environment of co-twins (Kendler et al. 1992a). Although a comprehensive treatise of putative environmental risk factors evaluated in a similar manner was beyond the scope of the present study, readers should note the relatively low power of latent variable models for identifying any but very sizeable effects of the familial environment shared by twins (Neale et al. 1994).

This work was supported by grants $\mathrm{MH}-45268, \mathrm{MH}-$ 40829, MH-49492, MH-01277 and RR-08123 from the National Institutes of Health, Bethesda, MD and grants from the Carman Trust and the MacArthur Network on Psychopathology.

\section{REFERENCES}

Akaike, H. (1987). Factor analysis and AIC. Psychometrika 52, 317-332.

American Psychiatric Association (1987). Diagnostic and Statistical Manual of Mental Disorders (3rd edition, revised) (DSM-III-R). APA: Washington, DC.

Andrade, L., Eaton, W. W. \& Chilcoat, H. (1994). Lifetime comorbidity of panic attacks and major depression in a population based study. Symptom profiles. British Journal of Psychiatry 165, 363-369.

Aneshensel, C. S., Estrada, A. L., Hansell, M. J. \& Clark, V. A. (1987). Social psychological aspects of reporting behavior: lifetime depressive episode report. Journal of Health and Social Behavior 28, 232-246.

Angst, J., Dobler-Mikola, A. \& Binder, J. (1984). The Zurich Study - a prospective epidemiological study of depressive, neurotic and psychosomatic syndromes. I. Problem, Methodology. European Archives of Psychiatric and Neurological Science 234, 13-20.

Bowlby, J. (1980). Attachment and Loss: Volume III: Loss. Sadness and Depression. Basic Books: London. 
Bromet, E. J., Dunn, L. O., Connell, M. M., Dew, M. A. \& Schulberg, H. C. (1986). Long-term reliability of diagnosing lifetime major depression in a community sample. Archives of General Psychiatry 43, 435-440.

Brown, C., Schulberg, H. C., Madonia, M. J., Shear, M. K. \& Houck, P. R. (1996). Treatment outcomes for primary care patients with major depression and lifetime anxiety disorders. American Journal of Psychiatry 153, 1293-1300.

Brown, G. W. \& Harris, T. O. (1978). Social Origins of Depression A Study of Psychiatric Disorder in Women. Tavistock: London.

Cannell, C. F. \& Fowler, F. J. (1963). A comparison of a selfenumerative procedure and a personal interview: a validity study. Public Opinion Quarterly 27, 250-264.

Carey, G. \& Gottesman, I. I. (1978). Reliability and validity of binary ratings: areas of common misunderstanding in diagnosis and symptom ratings. Archives of General Psychiatry $\mathbf{3 5}$, 1454-1459.

Cohen, J. (1960). A coefficient of agreement for nominal scales. Educational and Psychological Measurement 20, 37-46.

Eaton, W. W., Kramer, M., Anthony, J. C., Dryman, A., Shapiro, S. \& Locke, B. Z. (1989). The incidence of specific DIS/DSM-II mental disorders: data from the NIMH Epidemiological Catchment Area Program. Acta Psychiatrica Scandinavica 79, 163-178.

Eaves, L. J., Eysenck, H. J. \& Martin, N. G. (1989). Genes, Culture and Personality: An Empirical Approach. Academic Press: San Diego.

Falconer, D. S. (1967). Introduction to Quantitative Genetics. Oliver \& Boyd: London.

Fendrich, M., Weissman, M. M., Warner, V. \& Mufson, L. (1990). Two-year recall of lifetime diagnoses in offspring at high and low risk for major depression: the stability of offspring reports. Archives of General Psychiatry 47, 1121-1127.

Goethe, J. W., Fischer, E. H. \& Wright, J. S. (1993). Severity as a key construct in depression. Journal of Nervous and Mental Disease 181, 718-724.

Helzer, J. E., Robins, L. N., Groughan, J. \& Welner, A. (1981). Renard Diagnostic Interview: its reliability and procedural validity with physicians and lay interviewers. Archives of General Psychiatry 38, 393-398.

Jorm, A. F., Duncan-Jones, P. \& Scott, R. (1989). An analysis of the re-test artefact in longitudinal studies of psychiatric symptoms and personality. Psychological Medicine 19, 487-493.

Keller, M. B., Klein, D. N. \& Hirschfeld, M. A. (1995). Results of the DSM-IV mood disorders field trial. American Journal of Psychiatry 152, 843-849.

Kendler, K. S. \& Gardner, Jr., C. O. (1998). The boundaries of major depression: an evaluation of DSM-IV criteria. American Journal of Psychiatry 155, 172-177.

Kendler, K. S., Neale, M. C., Kessler, R. C., Heath, A. C. \& Eaves, L. J. (1992a). Childhood parental loss and adult psychopathology in women: a twin study perspective. Archives of General Psychiatry 49, 109-116.

Kendler, K. S., Neale, M. C., Kessler, R. C., Heath, A. C. \& Eaves, L. J. (1992b). Major depression and generalized anxiety disorder. Same genes, (partly) different environments? Archives of General Psychiatry 49, 716-722.

Kendler, K. S., Neale, M. C., Kessler, R. C., Heath, A. C. \& Eaves, L. J. (1993a). The lifetime history of major depression. Reliability of diagnosis and heritability. Archives of General Psychiatry 50, 863-870.

Kendler, K. S., Neale, M. C., Kessler, R. C., Heath, A. C. \& Eaves, L. J. (1994). Parental treatment and the equal environment assumption in twin studies of psychiatric illness. Psychological Medicine 24, 579-590.

MacClean, C. J. (1988). Assessing changes in risk factor effect over multiple levels of severity. American Journal of Epidemiology 127, 663-673.

McGuffin, P., Katz, R., Watkins, S. \& Rutherford, J. (1996). A hospital based twin register of the heritability of DSM-IV unipolar depression. Archives of General Psychiatry 53, 129-136.
Neale, M. C. (1997). Mx: Statistical Modeling, Fourth Edition. Department of Psychiatry, Box 710, MCV, Richmond, VA 23298.

Neale, M. C. \& Cardon, L. R. (1992). Methodology for Genetic Studies of Twins and Families. Kluwer Academic Publishers: The Netherlands.

Neale, M. C. \& Kendler, K. S. (1995). Models of comorbidity for multifactorial disorders. American Journal of Human Genetics 57, 935-953.

Neale, M. C., Eaves, L. J. \& Kendler, K. S. (1994). The power of the classical twin study to resolve variation in threshold traits. Behavior Genetics 24, 239-258.

Pini, S., Goldstein, R. B., Wickramaratne, P. J. \& Weissman, M. M. (1994). Phenomenology of panic disorder and major depression in a family study. Journal of Affective Disorders 30, 257-72.

Prusoff, B. A., Merikangas, K. R. \& Weissman, M. M. (1988). Lifetime prevalence and age of onset of psychiatric disorders recall 4 years later. Journal of Psychiatric Research 22, 107-117.

Reich, J., Warshaw, M., Peterson, L. G., White, K., Keller, M., Lavori, P. \& Yonkers, K. A. (1993). Comorbidity of panic and major depressive disorder. Journal of Psychiatric Research 27, (suppl. 1), 23-33.

Rice, J. P., Reich, T., Anderson, N. C., Lavori, P. W., Endicott, J., Clayton, P. J., Keller, M. B., Hirschfeld, R. M. A. \& Klerman, G. L. (1984). Sex-related differences in depression. Journal of Affective Disorders 71, 199-210.

Rice, J. P., Rochberg, N., Endicott, J., Lavori, P. W., Miller, C. (1992). Stability of psychiatric diagnoses: an application to the affective disorders. Archives of General Psychiatry 49, 824-830.

Robins, E. \& Guze, S. B. (1970). Establishment of diagnostic validity in psychiatric illness: its application to schizophrenia. American Journal of Psychiatry 126, 107-111.

Roy, A., Neale, M. C., Pedersen, N. L., Mathe, A. A. \& Kendler, K. S. (1995). A twin study of generalized anxiety disorder and major depression. Psychological Medicine 25, 1037-1049.

Rubio-Stipec, M., Freeman, Jr D. H., Robins, L., Shrout, P., Canino, G. \& Bravo, M. (1992). Response error and the estimation of lifetime prevalence and incidence of alcoholism: experience in a community survey. International Journal of Methods in Psychiatric Research 2, 217-224.

SAS Institute Inc. (1990). SAS/STAT Users Guide, Version 6, Fourth Edition. SAS Institute Inc.: Cary, NC.

Sobin, C., Weissman, M. M., Goldstein, R. B., Adams, P., Wickramaratne, P., Warner, V. \& Lish, J. D. (1993). Diagnostic interviewing for family studies: comparing telephone and face-toface methods for the diagnosis of lifetime psychiatric disorders. Psychiatric Genetics 3, 227-233.

Spence, J. E., Corey, L. A., Nance, W. E., Marazita, M. L., Kendler, K. S. \& Schieken, R. M. (1988). Molecular analysis of twin zygosity using VNTR DNA probes. American Journal of Human Genetics 43, A159 (Abstract).

Spitzer, R. L., Williams, J. B. \& Gibbon, M. (1987). Structured Clinical Interview for DSM-III-R. Biometrics Research Division, New York State Psychiatric Institute: New York.

Spitzer, R. L., Endicott, J. \& Robins, E. (1975). Research Diagnostic Criteria $(R D C)$ for a Selected Group of Functional Disorders. Biometrics Research Division, New York State Psychiatric Institute: New York.

Wainwright, N. W. J., Surtees, P. G. \& Gilks, W. R. (1997). Diagnostic boundaries, reasoning and depressive disorder, I. Development of a probabilistic morbidity model for public health psychiatry. Psychological Medicine 27, 835-845.

Weeks, M. F., Kulka, R. A., Lessler, J. T. \& Whitmore, R. W. (1983). Personal versus telephone surveys for collecting household health data at the local level. American Journal of Public Health 73, 1389-1394.

Weissman, M. M., Bruce, M. L., Leaf, P. J., Florio, L. P. \& Holzer, C. (1991). Affective disorders. In Psychiatric Disorders in America: the Epidemiologic Catchment Area Study (ed. L. N. Robins, and D. A. Regier), pp. 53-80. Free Press: New York.

Wells, K. B., Burnam, M. A., Leake, B. \& Robins, L. N. (1988). 
Agreement between face-to-face and telephone-administered versions of the depression section of the NIMH Diagnostic Interview Schedule. Journal of Psychiatric Research 22, 207-220.

Wilhelm, K. \& Parker, G. (1994). Sex differences in lifetime depression rates: fact or artefact? Psychological Medicine 24, 97-111.

Wilhelm, K., Parker, G. \& Hadzi-Pavlovic, D. (1997). Fifteen years on: evolving ideas in researching sex differences in depression. Psychological Medicine 27, 875-883.
Williams, J. B. W., Gibbon, M., First, M. B., Spitzer, R. L., Davies, M., Borus, J., Howes, M. J., Kane, J., Pope, Jr. H. G., Rounsaville, B. \& Wittchen, H-U. (1992). The structured clinical interview for DSM-III-R (SCID) 11. Multisite test-retest reliability. Archives of General Psychiatry 49, 630-636.

Williams, L. J. \& Holahan, P. J. (1994). Parsimony-based fit indices for multiple indicator models: do they work? Structural Equation Modeling 1, 161-189. 